\title{
Implementation of the Patient Self-Determination Act: A Comparison of Nursing Homes to Hospitals
}

\author{
Melissa D. Zwahr \\ Pennsylvania State University \\ Denise C. Park \\ University of Michigan \\ Thomas A. Eaton \\ Edward J. Larson \\ University of Georgia
}

The Patient Self-Determination Act (PSDA) requires all health care facilities receiving Medicare or Medicaid funds to provide information about advance directives and the right of patients to refuse medical treatment. Administrators of 155 nursing homes in the state of Georgia completed surveys to assess implementation practices employed to comply with the PSDA mandates, knowledge of the law, and perceived effects of the passage of the PSDA. Responses from nursing home administrators were summarized, reported, and compared to results obtained from Georgia hospitals. Important differences were discovered. Nursing homes routinely provide more types of information to residents and spend more time with residents explaining relevant information than do hospitals, but hospital administrators demonstrated better knowledge of the PSDA and state law than nursing home counterparts. The implications of findings regarding the implementation of the PSDA and its overall effectiveness are discussed.

The Patient Self-Determination Act (PSDA) (Omnibus Budget Reconciliation Act of 1990) was passed by the U.S. Congress in November 1990. It requires all health care facilities receiving Medicare or Medicaid funds to provide patients - at the time of admission — with written information about

\footnotetext{
AUTHORS' NOTE: This research was supported by a grant to the last three authors from the AARP Andrus Foundation, Washington, D.C. The authors thank John Gerks for his assistance with data analysis. Correspondence concerning this article should be addressed to Melissa D. Zwahr, Gerontology Center, 105 Henderson Building South, The Pennsylvania State University, University Park, PA, 16802.
} 
advance directives and the right of patients to refuse medical treatment. Advance directives include living wills and durable powers of attorney for health care (DPAHC). Generally, living wills stipulate a person's desires for the withholding of specific medical treatments and interventions, whereas DPAHCs appoint a proxy to make decisions when the principal is unable to make them. Both types of documents reflect a person's preferences regarding medical treatment in a future instance when the person is unable to state these treatment preferences for themselves-such as if the patient enters a persistent, vegetative state. One of the major goals associated with the PSDA was to encourage adults to complete advance directives so that their end-of-life treatment preferences could be carried out (Wolfe et al., 1991). The PSDA legislation was premised on the belief that more people would execute medical advance directives if they were better informed of their right to do so (Park, Eaton, Larson, \& Palmer, 1994).

Proponents of the PSDA were concerned that many health care facilities would only implement a minimal paper compliance with the mandates of the law by simply dispensing written information about patient rights rather than providing supportive, interactive communication to enhance patient decision making (Cate \& Gill, 1991; LaPuma, Orentlicker, \& Moss, 1991; Lynn \& Teno, 1993; McCloskey, 1991; Wolfe et al., 1991). Legislators recognized that the success of the PSDA legislation depended on implementation practices at the institutional level. However, because this legislation applies to a variety of health care facilities that service widely varying medical consumers and have different operating characteristics, it is conceivable that the PSDA may be implemented differently in the various settings. For example, hospitals typically admit a heterogenous mixture of patients of all ages, that require a variety of services ranging from those having an elective procedure to improve quality of life to those requiring a critical, lifesaving intervention. The vast majority of patients admitted have the capacity to make important treatment decisions. In contrast, nursing homes typically admit a more homogenous population of frail elderly who are not in an acute state but who are less likely to be able to make decisions about their health care. Because of the different patient profiles, there may be differences in the procedures used by various types of facilities to provide patients with information about advance directives, as mandated by the PSDA.

Early studies addressed implementation practices in the hospital setting. Park et al. (1994) examined how 81 Georgia hospitals implemented the mandates of the PSDA. They found that, typically, Georgia hospitals adopted a minimalist implementation. Specifically, results indicated that patients usually receive written materials explaining medical advance directives at the time of admission but little or no effort is made to supplement the written 
materials with verbal explanations or other types of interactive communication. The results of Park et al. may not generalize to other health care facilities, such as nursing homes. What provisions, if any, are made by nursing homes with regard to informing patients with declining health, cognitive impairment, interpersonal deficits, or combinations of the above about advance directives (Altman \& Parmelee, 1992)?

To determine the implementation practices used in nursing homes, a statewide survey of nursing home institutions was conducted in Georgia. The surveys were sent only to facilities in the state of Georgia because legislation regarding advance directives varies from state to state (Eaton \& Larson, 1991) and one dimension of the survey measured knowledge of relevant state law. However, Georgia, like most other states, recognizes the legality of the two most common types of advance directives-the living will and the DPAHC. Georgia's living will legislation (passed in 1984) and the Durable Power of Attorney for Health Care Act (passed in 1990) were both amended in 1992, consequent to the passage of the PSDA. Thus, although the survey was limited to Georgia facilities, the results undoubtedly reflect attitudes, problems, and experiences encountered by facilities in other states as well.

Results obtained from the nursing home sample were compared to results obtained from the Georgia hospital sample (Park et al., 1994) so that a direct comparison was made of implementation practices employed in the two types of facilities. Some basic issues addressed concerned implementation procedures, knowledge of the mandates of the PSDA and Georgia law regarding advance directives, perceived effects of the passage of the law, and perceived attitudes of various personnel and patients toward the PSDA legislation.

\section{Method}

\section{Subjects: Nursing Home Characteristics}

Administrators of every nursing home in the state of Georgia $(N=355)$ were sent a survey assessing their implementation practices regarding the PSDA. Of the nursing homes receiving surveys, 155 nursing homes returned completed surveys for a response rate of $44 \%$. Administrators receiving the surveys were instructed to complete them or give them to an appropriate person for completion. Most of those completing the surveys were classified as administrative and/or executive directors $(74 \%)$ or were directors of individual departments (13\%). Nursing homes responding to the survey were owned by a variety of investor organizations including corporate chains $(49 \%)$, a hospital authority (10\%), stand-alone organizations ( $23 \%)$, or other 
types of organizations, such as nonprofit organizations (18\%). Additionally, $13 \%$ of the nursing home respondents indicated that their nursing homes were administratively linked with a hospital.

As in Park et al. (1994), the nursing homes were divided along two dimensions. First, a median split procedure was used to classify the facilities as large or small, based on the number of licensed beds the facility contained. Thus, 74 nursing homes were classified as large (contained more than 100 beds), whereas 81 nursing homes were classified as small (contained 100 beds or less). Second, the facilities were categorized as urban or rural based on whether they were located in Metropolitan Statistical Areas (MSAs). Of the nursing homes surveyed, 61 were located in MSAs whereas 94 were located in rural areas. Distinctions between responses obtained from large/small and urban/rural facilities are made only when comparisons revealed significant differences.

\section{Procedure}

The survey instrument was mailed to nursing home administrators by the University of Georgia Survey Research Center in October 1992, approximately 10 months after the PSDA took effect in December 1991. Institutions not completing the surveys received follow-up phone calls from the University of Georgia Survey Research Center, and all surveys were returned by December 1992.

The instrument sent to nursing home administrators was virtually identical to the instrument mailed to hospital administrators, as described in Park et al. (1994). The instrument consisted of 62 questions that were mostly forced choice. For the majority of questions, respondents were given a number of items to choose from and asked to select the item(s) that best described their institutions. However, 13 of the items were short-answer, open-ended questions requiring a brief explanation (e.g., What is the size of your facility?). Responses to these questions were summarized and categorized across respondents and were subsequently coded for data analysis. Finally, two additional forced-choice questions were added to the nursing home instrument that did not appear on the hospital survey: (a) Is the nursing home administratively linked with a hospital? and (b) For what level of care is the nursing home certified?

The items contained in the survey assessed five different aspects of the PSDA. First, a number of questions evaluated the process by which facilities became aware of the PSDA and how they formulated plans to comply with the legislation. Second, respondents were asked about the implementation process for the PSDA legislation at the institutional level and about mecha- 
nisms in place for ensuring patient comprehension. Third, the knowledge facility personnel had about the PSDA and associated state laws was evaluated. Fourth, the perceptions of facility personnel regarding the effects of the PSDA were assessed. Finally, the perceived attitudes of facility personnel and residents toward the PSDA legislation were evaluated.

\section{Results and Discussion}

\section{Awareness of the Law and Formulation of Policy}

After the PSDA was passed in November 1990, health care facilities had approximately 1 year to become aware of the legislation and develop plans to comply with the mandates of the law. Nursing homes in the state of Georgia were slow to develop an awareness of the passage of the PSDA and most did not find out about the law until 6 months after its passage. In fact, only $34 \%$ of nursing homes were aware of the legislation within 6 months of its passage. Nursing home administrators indicated heavy reliance on professional organizations, such as the Georgia Nursing Home Association (GNHA), for developing awareness of the passage of the PSDA and for formulation of plans for compliance. In fact, $46 \%$ of nursing homes reported first finding out about the PSDA from GNHA, and $40 \%$ of nursing homes responded that they found out about the legislation through multiple sources that might include GNHA. Furthermore, $74 \%$ of nursing homes listed GNHA as the primary source of information regarding the formulation of plans for compliance with the mandates of the PSDA. These results parallel those reported by Park et al. (1994) from Georgia hospitals. Only 39\% of hospitals were aware of the PSDA legislation within 6 months of its passage, and hospitals indicated similar reliance on their primary professional organization, the Georgia Hospital Association, in developing awareness of the law and in formulating compliance policies. Taken together, these findings support the widely held belief about the role of professional organizations in shaping institutions' responses to new developments in the law (Givelber, Bowers, \& Blitch, 1984; Wiley, 1981).

\section{Implementation of the PSDA}

Questions were included in the survey of Georgia nursing homes to ascertain how facilities complied with the mandates of the PSDA-that is, what steps were taken to inform patients about advance directives and to ensure that patients comprehended their rights under the law? 
Type of information provided. A major concern of proponents of the PSDA was that health care facilities would offer only written materials at the time of admission and would fail to actively engage patients in a decision-making process geared toward enhancing patient autonomy. Although the majority of nursing homes surveyed provide written materials prepared either by the institutions $(62 \%)$ and/or by others (45\%), many nursing homes also provide supplemental information to their patients without special request. When compared to results obtained by Park et al. (1994), procedures followed by Georgia nursing homes are more consonant with the original spirit of the PSDA than are procedures routinely followed by hospitals. Nursing homes are more likely than hospitals to provide verbal explanations of relevant laws (79\% vs. 33\%), routine follow-up consultations (32\% vs. 5\%), forms for living wills ( $42 \%$ vs. $25 \%$ ), and forms for DPAHCs ( $45 \%$ vs. $27 \%$ ).

Amount of time spent explaining information. Another index of how vigilantly health care facilities actively educate medical consumers of their rights under the PSDA is the amount of time spent explaining relevant state laws. Most nursing homes (73\%) reported spending more than 10 minutes explaining patient rights under the PSDA. In fact, $18 \%$ reported spending 21 to 30 minutes explaining advance directive information, and $19 \%$ reported spending more than 30 minutes. When compared to the amount of time spent by hospitals (Park et al., 1994), chi-square analyses revealed a significant difference as a function of facility in the amount of time spent with residents and/or patients, $\chi^{2}(6)=116.11, p<.001$. Nursing homes were more likely to spend longer periods of time with patients than were hospitals.

Ability of residents and/or patients to comprehend information. The type of information provided, as well as the amount of time spent explaining it, may vary as a function of the characteristics of the patients or residents to which it is presented. Respondents were asked to specify the percentage of residents perceived as sufficiently mentally competent to be given information about advance directives and able to execute advance directives on their own. Results indicated that an estimated $31 \%$ of nursing home residents were able to comprehend pertinent advance directive materials and make independent decisions, whereas Park et al. (1994) reported that respondents from hospitals indicated that $74 \%$ of their patients were able to do so. A 2 (facility) $\times 2$ (size) ANOVA indicated that the main effect of facility was statistically significant, $F(7,211)=199.94, p<.01$. Additionally, a main effect for size was obtained with large nursing homes and hospitals reporting a greater percentage of residents and/or patients able to understand information and 
execute advance directives than did smaller facilities, $57.75 \%$ and $47.48 \%$, respectively, $F(7,211)=11.91, p<.01$.

Dissemination of information. The majority of nursing homes surveyed (74\%) reported that information regarding advance directives and the right to refuse medical treatment was most commonly presented during admissions. Similarly, Park et al. (1994) found that $83 \%$ of hospitals disseminated information at this time. However, whereas $72 \%$ of nursing home respondents indicated that members of the social services staff generally informed patients in the nursing home setting, in $83 \%$ of the hospitals participating in the survey this duty fell to the admissions staff. The differences in staff utilization, revealed by $t$ tests, were significant: Nursing homes were significantly more likely to use social services staff in information dissemination, $t(154)=12.92, p<.01$, whereas hospitals more frequently employed admissions staff, $t(154)=-13.29, p<.01$.

Maintenance of written records. One of the mandates of the PSDA is that health care facilities keep written records of patients' execution of advance care documents. Of nursing homes surveyed, $59 \%$ indicated that they maintained records regarding the number of patients who execute advance directives. Park et al. (1994) reported that only $19 \%$ of the Georgia hospitals surveyed did so.

In sum, the results of the present study indicate that implementation practices with regard to the PSDA in nursing homes are somewhat dissimilar from those in hospitals (Park et al., 1994). Nursing home procedures appeared to provide more opportunity for an active decision-making process to develop than do procedures implemented by hospitals. In fact, respondents from nursing homes reported spending longer amounts of time educating residents about advance directives and used more types of information in the process. There are a number of reasons that may account for these discrepancies in implementation practices. First, nursing home respondents estimated a smaller percentage of their patients to be able to understand the legal information presented to them and to make independent decisions regarding advance directives. The longer time period and more diverse materials may be necessary to convey information to a potentially mentally compromised population.

Second, nursing homes are heavily regulated and come under intense legal scrutiny. In fact, the regulations that apply to nursing homes are quite different from those applicable to hospitals (Johnson, 1991). According to Johnson (1991), enforcement of advance care directives is a primary concern 
in nursing homes, and administrators are particularly wary of any actionsuch as controversial withdrawal of treatment - that may incite investigation. Consequently, nursing homes, more so than hospitals, may express "a de facto requirement of a written advance directive prior to decisions to discontinue or forgo treatment" (Johnson, 1991, p. S4). The implementation practices followed by nursing homes, outlined here, are consistent with the policies expressed by Johnson.

Third, although both hospitals and nursing homes tend to disseminate information during admissions, the admissions processes in the two facilities are vastly different. Specifically, people entering hospitals are more likely to be in a critical or acute situation than those entering nursing homes. There may be insufficient time to elaborately explain rights under the PSDA during hospital admissions given the large number of admissions that hospitals routinely handle. Additionally, admission into a nursing home is typically an orientation process that may extend over a period of days in which the incoming resident is undergoing a significant change in lifestyle and may be preparing for permanent residence in the institution. Also, there is more likely to be sufficient time to explain advance directive information during the extended period of nursing home admission than is found during hospital admission. Family members of the incoming resident may also be involved in the proceedings and their questions may invoke a more extended discussion, thereby lengthening the information dissemination process in nursing homes.

\section{Knowledge of the Law}

Eleven questions were included in the surveys sent to Georgia nursing homes to determine whether the survey respondent understood the mandates of the PSDA and the state law. Analyses of survey respondents' performance on the questions about the PSDA and Georgia state law indicated that nursing home administrators accurately answered $73 \%$ of questions asked. The mean number correct responses out of 11 was $7.97(S D=1.58)$ across the nursing home respondents surveyed. Hospital respondents answered a mean of 8.95 $(S D=1.86)$ questions correctly (Park et al., 1994). To compare knowledge in nursing home respondents to hospital respondents, a 2 (facility) $\times 2$ (size) $\times 2$ (location) ANOVA was conducted on the mean knowledge scores. The following results were obtained: (a) a significant main effect for facility, with hospital respondents outperforming nursing home respondents, $F(7,228)=$ $10.99, p<.01$; (b) a significant main effect for size, with respondents from large facilities exhibiting more knowledge about the PSDA than respondents 
Table 1. Knowledge of PSDA as a Function of Facility, Size, and Location

\begin{tabular}{lll}
\hline & Small & Large \\
\hline $\begin{array}{lll}\text { Nursing homes } \\
\text { Urban }\end{array}$ & $8.00(1.29)$ & $7.85(1.53)$ \\
& $N=13$ & $N=48$ \\
Rural & $8.01(1.77)$ & $8.19(1.33)$ \\
& $N=68$ & $N=26$ \\
Hospitals & & \\
Urban & $7.00(1.58)$ & $9.06(1.30)$ \\
Rural & $N=5$ & $N=17$ \\
& $8.17(1.87)$ & $9.67(1.55)$ \\
\hline
\end{tabular}

NOTE: PSDA = Patient Self-Determination Act. Mean correct out of 11. Standard deviations in parentheses.

from small facilities, $F(7,228)=4.60, p<.05$; (c) a significant main effect for location, with respondents from rural facilities outperforming those from urban facilities, $F(7,228)=3.88, p=.05$; and (d) a significant facility by size interaction, in which post hoc $t$ tests revealed that respondents from large hospitals outperformed respondents from other types of facilities, $F(7,228)=$ $9.13, p<.01$. Table 1 depicts the mean knowledge scores as a function of facility, size, and location.

Although respondents from hospitals displayed better knowledge of the PSDA than respondents from nursing homes, and respondents from large facilities were more knowledgeable than respondents from smaller facilities, the percentage of questions answered correctly only ranged from $72 \%$ in large nursing homes to $86 \%$ in large hospitals. These results indicate that educators themselves have less than accurate knowledge of the law. Perhaps more attention needs to be focused on educating the educators, such that the original premises associated with the PSDA are not jeopardized by inaccurate communication.

\section{Perceived Effects of the PSDA}

Two questions were asked to determine the percentage of residents with advance directives in effect-both prior to the passage of the PSDA and subsequent to it. Prior to the passage of the PSDA, respondents from nursing homes estimated that $8 \%$ of their residents had effective advance directives. However, after the passage of the PSDA, nursing homes reported an increase in the percentage of residents with effective advance directives to an esti- 
mated $27 \%$. These numbers are higher than the numbers reported by Park et al. (1994). Park et al. found that an estimated 3\% of hospital patients had effective documents prior to the passage of the PSDA and $12 \%$ had them after the passage of the law. These differences, revealed by $t$ tests, were statistically significant: Nursing homes had a larger number of residents with advance directives in effect prior to the PSDA, $t(133)=2.08, p<.05$, as well as subsequent to its passage, $t(137)=3.79, p<.01$.

Additionally, the respondents from nursing homes were asked to specify whether residents generally executed advance directives prior to admission, at the time of admission, during their stay, or after discharge. Results indicated that residents of nursing homes typically execute advance directives either at the time of admission (35\%) or during their stay in the facility $(31 \%)$. In contrast, $40 \%$ of hospital patients completed advance directives prior to admission (Park et al., 1994). A chi-square analysis revealed significant differences in when documents were executed as a function of facility, $\chi^{2}(4)=78.79, p<.001$. Post hoc $t$ tests indicated that residents of nursing homes were more likely to execute advance directives at the time of admission or during their stay, whereas hospital patients were more likely to complete the documents prior to admission. Furthermore, $3 \%$ of hospital respondents indicated that patients executed advance directives after discharge, whereas none of the nursing home respondents answered in such a manner.

In sum, the proponents of the PSDA hypothesized that people did not execute advance directives because of a lack of information, and that distribution of information would encourage people to execute advance care documents. Results of this study, and those of Park et al. (1994), address this supposition. First, a proportional increase in the number of people who execute advance directives was observed subsequent to the passage of the PSDA in both nursing home residents and hospital patients. However, the absolute increase was, perhaps, not as large as the supporters of the PSDA might have anticipated. In hospitals, an estimated $9 \%$ more patients executed advance directives after the passage of the PSDA, whereas in nursing homes, there was an increase of approximately $19 \%$. As Park et al. pointed out, these relatively small increases raise the question of whether the PSDA is meeting its original objective-to increase use of medical advance directives. Recent research conducted by High (1993) indicates that even with intervention (e.g., intensive educational efforts and assistance in completing forms), increases in the number of people executing advance directives are relatively small due to their preference for family involvement in the decision-making process. The results of our study, as well as those of Park et al. and High, indicate the 
need for more research on understanding people's reluctance to formally execute advance directives.

Second, the increase in the number of persons executing advance directives, small though it was, may not be directly attributable to the passage of the PSDA but to an increase in general public awareness about advance directives. Although a large percentage of people in nursing homes execute advance directives at the time of admission or during their stay (after being presented with the information), those entering the hospital tend to execute directives prior to admission (before presentation of information). Other research supports this notion of heightened awareness regarding medical advance directives (e.g., Emanuel, Weinberg, Gonin, Hummel, \& Emanuel, 1993; Robinson, de Haven, \& Koch, 1993). However, this evident increase in public awareness may be a consequence of the passage of the PSDA legislation.

Third, nursing home residents were more likely than hospital patients to have advance directives - both prior to, and subsequent to, the passage of the PSDA. Possession of advance directives may be of more importance in nursing homes and in the best interest of nursing home facilities due to the extended stays (often permanent) of most nursing home residents, as well as the likelihood of residents facing infirmities and serious disease. Furthermore, whereas the vast majority of nursing home residents are elderly, a significant portion of hospital patients are relatively young. Younger persons may not be inclined to learn about or to consider an advance directive because of their unwillingness to consider end-of-life issues-a remote prospect for most young people.

\section{Problems and Attitudes Regarding the PSDA}

Finally, questions were included in the survey that addressed problems and attitudes regarding the PSDA. Areas of interest included (a) the major problems encountered by nursing homes in conforming to the mandates specified by the law, (b) the role that facilities perceive for themselves regarding the PSDA, and (c) the attitudes of various personnel and residents toward the PSDA.

Major problems with compliance. Nursing home administrators were asked to characterize the major problems encountered in complying with the mandates of the PSDA. When asked to select the single most common problem encountered, nursing homes reported the difficulty in conveying the information in a manner that was easily understood by residents. In contrast, 
Table 2. Most Significant Problems Encountered During Implementation of PSDA

Nursing Homes $(\mathbf{N}=155)$ Hospitals $(\mathrm{N}=81)$

\begin{tabular}{lll}
\hline Admission is inappropriate time & 43 & 86 \\
Takes too much time & 17 & 38 \\
Staff not qualified to convey information & 32 & 38 \\
Insufficient materials to develop plan & 17 & 01 \\
Insufficient time to develop plan & 17 & 14 \\
Difficult to convey information to & & \\
$\quad$ residents/patients & 74 & 74 \\
\hline
\end{tabular}

NOTE: PSDA = Patient Self-Determination Act. Numbers represent percentage responding when asked to select top three choices.

Park et al. (1994) reported that hospitals found their number one problem to be working the presentation of advance directive information into the admission process. Table 2 summarizes problems reported by nursing homes and hospitals in complying with the PSDA legislation when they were allowed to make three choices.

Role of facility. Respondents from nursing homes were asked to select a statement that best summarized their perception of the role of their facility with respect to the PSDA from among a number of choices: (a) disseminate information about advance directives, (b) actively educate residents and/or patients with respect to advance directives, (c) encourage residents and/or patients to execute advance directives at the time of admission, and (d) encourage residents and/or patients to execute advance directives after discharge. Results indicated that $52 \%$ of nursing home respondents perceived their role to be active educators of PSDA mandates. In contrast, Park et al. (1994) reported that hospitals perceived their role as being disseminators of information (52\%). A chi-square analysis and post hoc $t$ tests revealed that these differences were significant, $\chi^{2}(3)=30.73, p<.001$. The results obtained from these questions are summarized in Table 3.

Attitudes of personnel and patients/residents toward PSDA. Persons completing the survey were asked to rate whether administrators, nursing staff, physicians, and social services staff strongly favored, favored, did not favor, or strongly did not favor this requirement, or whether opinion was about even. Generally, nursing home personnel supported the PSDA; $68 \%$ of administrators, $66 \%$ of nursing staff, $57 \%$ of physicians, and $80 \%$ of social services staff 
Table 3. Role of Facility Regarding PSDA

\begin{tabular}{lccc}
\hline & $\begin{array}{c}\text { Nursing Homes } \\
(\mathrm{N}=155)\end{array}$ & $\begin{array}{c}\text { Hospitals } \\
(\mathbf{N}=81)\end{array}$ & $\mathbf{t}$ \\
\hline Disseminate information & 26 & 52 & $3.99^{*}$ \\
Actively educate & 52 & 25 & $4.10^{*}$ \\
Encourage execution during stay & 01 & 00 & $2.35^{*}$ \\
Encourage execution after discharge & 01 & 06 & $2.59^{*}$ \\
\hline
\end{tabular}

NOTE: PSDA = Patient Self-Determination Act. Numbers represent percentage responding.

* Significant at $p<.01$.

were rated as either favoring or strongly favoring the mandates of the legislation. A 2 (facility) $\times 4$ (personnel) repeated measures ANOVA was conducted to compare attitudes of nursing home personnel to those of hospitals. This analysis revealed a significant main effect for facility, $F(1$, $171)=6.09, p<.01$, with nursing home personnel rated as favoring the PSDA more than hospital personnel, as well as a significant main effect of personnel, $F(3,513)=7.85, p<.01$. Pair-wise comparisons among the four types of personnel indicated that the social services staff were rated as favoring the PSDA more than any other type of personnel-there were no differences among the ratings of any other personnel types. Social services personnel showed more favorable ratings than did administrators, $F(1,203)=23.18, p<$ .01 , nursing staff, $F(1,206)=4.94, p<.05$, and physicians, $F(1,188)=8.72$, $p<.01$. The facility by personnel interaction failed to reach significance.

The administrators of the nursing homes surveyed were also asked to summarize residents' opinions about being informed of their rights under the PSDA. Overall, the residents of nursing homes were rated as generally in favor of being presented with this information. Specifically, 59\% of nursing home administrators rated their residents as either strongly in favor of, or in favor of, being informed of their rights. Comparative analyses with results obtained from the Park et al. (1994) hospital sample indicated that nursing home residents were perceived to be more in favor of being informed of their rights under the PSDA than were hospital patients. Of hospital administrators, $33 \%$ rated their patients as in favor of or strongly in favor of being informed, and $43 \%$ estimated that their patients were about even in their opinions of being informed about their rights to advance directives. Very few respondents from either nursing homes or hospitals indicated that their residents or patients were not in favor of the PSDA mandates. Chi-square analyses conducted on these questions indicated a significant difference in perceptions 
of residents' and/or patients' receptivity to information about treatment directives, $\chi^{2}(5)=17.36, p<.01$. Nursing home residents were more likely to be perceived as being strongly in favor of PSDA mandates than hospital patients, whereas hospital patients were more likely to be perceived as having an even opinion than were nursing home residents.

In sum, the results of this investigation indicated that (a) nursing homes and hospitals had some similar problems in complying with the mandates of the PSDA, (b) the two types of facilities had different perceptions of their roles regarding the legislation, and (c) personnel and residents or patients were generally supportive of the law. First, with regard to problems encountered in complying with the mandates of the PSDA, both nursing homes (74\%) and hospitals (74\%) listed the difficulty of conveying information in a manner that was easily understood by residents and/or patients as a major problem in complying with the PSDA legislation. Interestingly, hospitals were more likely than nursing homes ( $38 \%$ vs. $17 \%$ ) to list "takes too much time" as a major implementation problem even though hospitals spent considerably less time than nursing homes with the process.

Hospital respondents (86\%), and to a lesser extent nursing home respondents $(43 \%)$, expressed the difficulty in conveying advance directive information during the admissions process. These findings may be a consequence of the volume of admissions typically processed by hospitals in comparison to nursing homes or, perhaps, more time than is allowed during admissions is necessary to adequately explain advance directive information. Perhaps, as suggested by Park et al. (1994), discussion of advance directive information should occur in some other location-such as a physician's office or when a driver's license is renewed.

Second, there were significant differences in perceived institutional roles and attitudes regarding the PSDA. Nursing homes perceive themselves as educators, whereas hospitals perceive themselves as providers of information. Moreover, nursing home personnel and residents are perceived to more strongly support the PSDA mandates. These differences in perception are not surprising. Hospital personnel may be biased against the PSDA because of their focus on medical intervention and the preservation of life. Nursing homes, however, may have a keener institutional interest in increasing the percentage of residents who execute advance directives, and nursing home residents may perceive more personal relevance in having such documents. As nursing home residents are more likely to face infirmities and disease progression during their stay, the advantages of nursing home residents having advance directives are twofold: (a) Advance directives inform families, as well as nursing home staff, about end-of-life treatment preferences; 
and (b) advance directives provide legal protection for nursing homes in the event of difficult and ethically challenging treatment decisions.

\section{Conclusion}

The results of this study and subsequent comparisons to results obtained by Park et al. (1994) identify some similarities in the implementation practices of Georgia nursing homes and hospitals regarding the PSDA legislation. Both hospitals and nursing homes were slow to learn of the PSDA and relied primarily on their respective professional organizations for devising strategies for compliance. Moreover, both nursing homes and hospitals report that one of the biggest challenges faced was devising a plan that effectively conveys information regarding advance directives to residents and/or patients with wide ranging differences in illness, intelligence, and mental capacity.

The differences in implementation practices of hospitals and nursing homes are, perhaps, more striking. When compared to hospitals, nursing homes are more likely to provide verbal explanations of written materials, offer routine follow-up, and actively facilitate execution of advance directives. The typical nursing home spends considerably more time with its residents in this process than does the typical hospital. The interactive implementation practices of nursing homes are more in line with the aspirations of the proponents of the PSDA than the minimalist practices of hospitals. Moreover, a greater percentage of nursing home residents execute advance directives than hospital patients.

\section{Implications of Findings}

The differences in implementation practices of hospitals and nursing homes shed light on the overall impact and effectiveness of the PSDA. Supporters of the PSDA might lament the minimal implementation practices of hospitals but be encouraged by the active educational practices of nursing homes. Yet, there are reasons to temper this enthusiasm with a degree of caution. For instance, although nursing homes spend more time with residents and employ more interactive techniques, they tend to know less than hospitals about the laws they are explaining.

Additionally, the limited increase in percentages of nursing home residents who execute advance directives suggests the limits of the PSDA legislation. Less than $30 \%$ of the nursing home population was estimated to have executed advance directives even after significant educational efforts. Thus, health care providers are likely to continue facing ethically and legally 
complex treatment decisions without clear guidance from patients or their designated agents.

Moreover, in this study, both nursing home and hospital respondents indicated less-than-desirable maintenance of written records regarding residents' and patients' possession of advance directives. Lackadaisical maintenance of records in these and other health care facilities may prohibit residents' and/or patients' end-of-life treatment preferences from being met and carried out. This is especially disturbing in light of recent research suggesting that even when advance directives have been executed and recorded, they are frequently not honored by the medical community (Schneiderman, Kronick, Kaplan, Anderson, \& Langer, 1992).

Given these considerations, legislators, policy makers, and health care professionals may need to rethink the entire advance directive process (High, 1993). Advocates of medical advance directives will need to consider these problems and identify strategies to (a) increase use of medical advance directives, and (b) ensure compliance with resident and patient wishes.

\section{Limitations}

There are several methodological issues that need to be considered when evaluating this study. First, the majority of respondents who completed both the nursing home and hospital surveys were administrators and/or executive directors. Unlike heads of individual departments, such as head of social services or head of admissions, administrators and executive directors are frequently removed from the day-to-day procedures regarding compliance with the PSDA. Because of this, administrators and executive directors may, for instance, over- or understate the amount of time spent explaining advance directive information to residents and/or patients, and may be less familiar with the dictates of the legislation and consequently perform more poorly when tested about knowledge of the law. Thus, some of the results should be interpreted with this in mind.

Second, although questions included in the survey addressed certain aspects of nursing home and hospital's procedures regarding provision of advance directive information, many questions are left unanswered. For instance, results revealed that incoming residents of nursing homes frequently execute advance directives during the admissions process. It may be less than ideal for nursing home residents to make this decision in the midst of the stressful admissions period. The effects of making important decisions during this time are currently unknown. As another example, the results of this study indicated that a large percentage of nursing homes reported spending more than 10 minutes explaining residents' rights under the PSDA. 
To whom was the discourse addressed? To patients alone, or to patients' families as well? Family members often play a vital role in end-of-life treatment decisions-an issue that was not addressed in the present study.

\section{References}

Altman, W. M., \& Parmelee, P. A. (1992). Discrimination based on age: The special case of the institutionalized aged. In D. K. Kagehiro \& W. S. Laufer (Eds.), Handbook of psychology and law. New York: Springer.

Cate, F. H., \& Gill, B. A. (1991). The Patient Self-Determination Act: Implementation issues and opportunities. Annenberg Washington Program, 1-25.

Eaton, T. A., \& Larson, E. J. (1991). Experimenting with the "right to die" in the laboratory of the states. Georgia Law Review, 25, 1253-1326.

Emanuel, E. J., Weinberg, D. S., Gonin, R., Hummel, L. R., \& Emanuel, L. L. (1993). How well is the Patient Self-Determination Act working? An early assessment. American Journal of Medicine, 95, 619-628.

Givelber, D. J., Bowers, W. J., \& Blitch C. L. (1984). Tarasoff, myth and reality: An empirical study of private law in action. Wisconsin Law Review, 84, 443-497.

High, D. M. (1993). Advance directives and the elderly: A study of intervention strategies to increase use. The Gerontologist, 33, 342-349.

Johnson, S. (1991). PSDA in the nursing home. Hastings Center Report, 2I(Suppl.), S3-S4.

LaPuma, J., Orentlicker, D., \& Moss, R. J. (1991). Advance directives on admission: Clinical implications and analysis of the Patient Self-Determination Act of 1990. Journal of the American Medical Association, 266, 402-405.

Lynn, J., \& Teno, J. M. (1993). After the Patient Self-Determination Act: The need for empirical research on formal advance directives. Hastings Center Report, 23, 20-24.

McCloskey, E. L. (1991). The spirit of the PSDA. Hastings Center Report, 21(Suppl.), S14-15.

Omnibus Budget Reconciliation Act of 1990, P.L. 101-508, 4206 and 4751, codified as 42 U.S.C. $1395 \mathrm{cc}$ (a) (1) (q), $1395 \mathrm{~mm}$ (c) (8), $1395 \mathrm{cc}$ (f), 1396a (57), (58), 1396a (w).

Park, D. C., Eaton, T. A., Larson, E. A., \& Palmer, H. T. (1994). Implementation and impact of the Patient Self-Determination Act. Southern Medical Journal, 87, 971-977.

Robinson, M. K., de Haven, M. J., \& Koch, K. A. (1993). Effects of the Patient Self-Determination Act on patient knowledge and behavior. The Journal of Family Practice, 37, 363-368.

Schneiderman, L. J., Kronick, R., Kaplan, R. M., Anderson, J. P., \& Langer, R. D. (1992). Effects of offering advance directives on medical treatments and costs. Annals of Internal Medicine, $117,599-606$.

Wiley, J. (1981). The impact of judicial decisions on professional conduct: An empirical study. Southern California Law Review, 55, 345-396.

Wolfe, S. M., Boyle, P., Callahan, D., Fins, J. J., Jennings, B., Nelson, J. L., Barondess, J. A., Brock, D. W., Dresser, R., Emanuel, L., Johnson, S., Lantos, J., Dacosta, R. M., Mezey, M., Orentlicher, D., \& Rouse, F. (1991). Sources of concern about the Patient Self-Determination Act. New England Journal of Medicine, 236, 1666-1671. 
Melissa D. Zwahr, Ph.D., is currently employed as a research associate in the Gerontology Center at Pennsylvania State University. Her primary research interests lie in the investigation of age-related changes in cognitive ability and the impact these changes have on everyday functioning - particularly in health-related domains. Her recent publications include "Age Differences in Inhibition: Possible Causes and Consequences" and "Mediators of Long-Term Memory Performance Across the Life Span."

Denise C. Park, Ph.D., is currently a professor of psychology and research scientist at the Institute of Gerontology at the University of Michigan. Her research interests are in how basic memory processes change with age, and also in practical applications of cognitive aging research to understand medication adherence, social functioning, and medical decision making. Titles of recent publications include "Mediators of Long-Term Memory Performance Across the Life Span" and "Remembering to Take Medications: The Importance of Memory Variables."

Thomas A. Eaton, J.D., is currently the J. Alton Hosch Professor at the University of Georgia School of Law. His primary research interests lie in health law and patient rights. Titles of recent publications include "A Profile of Tort Litigation in Georgia and Reflections on Tort Reform" and "The Limits of Advance Directives: A History and Assessment of the Patient Self-Determination Act."

Edward J. Larson, Ph.D., J.D., is currently a professor in the School of Law and History at the University of Georgia. His research interests lie in health-and medical-related legal issues. Titles of recent books include Sex, Race, and Science: Eugenics in the Deep South and Euthanasia: Spiritual, Medical, and Legal Issues in Terminal Health Care. 